\title{
Cuidando de quem cuida: projeto de intervenção como estratégia de aprendizagem no internato em medicina
}

\author{
Caring for caregivers: encouraging intervention activities as a learning strategy in medical internship
}

\author{
Ana Vitória Teixeira de Medeiros Gomes ${ }^{1}$ (D) | vitória_medeiros@hotmail.com \\ Erika Denise de Vasconcelos Florentino' (D) erikadenisevasconcelos@gmail.com \\ Myrlla Soares de Oliveira' ${ }^{1}$ (D) myrlla.soares@gmail.com \\ Thaiany Rego Silva' (1) rego.thaiany@gmail.com \\ Jucimara Alves de Souza² (1) jucimara_alves@hotmail.com
}

\begin{abstract}
RESUMO
Introdução: Este trabalho descreve a experiência de estruturação de um grupo terapêutico com cuidadores informais que apresentavam sinais de sobrecarga e que atuavam na prestação de cuidados a pessoas acamadas acompanhadas em visita domiciliar pela equipe de saúde da Estratégia de Saúde da Família (ESF).

Relato de Experiência: O grupo foi estruturado e desenvolvido por duas alunas do período de Internato do curso de Medicina no rodízio de Medicina de Família e Comunidade (MFC) junto a médica residente do segundo ano do Programa de Especialização em MFC, a preceptora do Programa e a enfermeira da equipe na unidade escola Josefa de Souza Silva, bairro Pedra Linda, em Petrolina-Pernambuco, a partir da identificação de um problema na comunidade e como parte do projeto de intervenção.

Discussão: Foram realizados sete encontros semanais com doze cuidadores, no período de maio a julho do ano de 2019. Cada encontro foi dividido em acolhida, apresentação da proposta do dia com a vivência de uma prática de autocuidado e finalizado com a sugestão de uma atividade para casa, a ser desenvolvida até o próximo encontro. Para o desenvolvimento das atividades foram utilizadas a abordagem comunitária e recursos como Educação Popular e Práticas Integrativas e Complementares em Saúde.

Conclusão: Foi observada a diminuição do estresse e sobrecarga nos cuidadores, a partir da prática do autocuidado e do fortalecimento das redes de apoio na comunidade. Paralelamente, foi percebida a potencialidade do projeto de intervenção como estratégia de aprendizado no processo de formação médica na ESF: as alunas puderam experimentar o estabelecimento da relação médico-paciente, o uso de tecnologias leves para o cuidado, a coordenação de um grupo terapêutico, compreender o significado de equidade, integralidade e longitudinalidade na prática da MFC, se reconhecerem como cuidadoras e a importância de cuidar de si para cuidar do outro.
\end{abstract}

Palavras-chave: Cuidadores; Atenção Primária a Saúde; Educação Médica; Terapias Complementares; Grupo Terapêutico.

\begin{abstract}
Introduction: This work describes the experience of structuring a therapeutic group with informal caregivers who showed signs of overload and who worked providing care to bedridden people followed through home visits by the health team of the Family Health Strategy (FHS).

Experience Report: The group was structured and developed by two students of the Internship period of the medical course during the rotation in Family and Community Medicine (FCM) together with the second-year resident physician of the Specialization Program in FCM, the preceptor of the Program, and the team nurse at the Josefa de Souza Silva school unit, Pedra Linda neighborhood, in Petrolina, state of Pernambuco, based on the identification of a problem in the community and as part of the intervention project.
\end{abstract}

Discussion: Seven weekly meetings were held with twelve caregivers, from May to July 2019. Each meeting was divided into welcoming activities, presentation of the day's proposal with the experience of a self-care practice and ended with the suggestion of a home activity, to be developed until the next meeting. For the development of activities, the community approach and resources such as Popular Education and Integrative and Complementary Practices in Health were used.

Conclusion: The reduction of stress and overload on caregivers was observed, from the practice of self-care and the strengthening of support networks in the community. At the same time, the potential of the intervention project was perceived as a learning strategy in the process of medical training in the FHS: the students were able to experience the establishment of the doctor-patient relationship, the use of light technologies for care, the coordination of a therapeutic group, understanding the meaning of equity, integrality and longitudinality in the practice of FCM, recognizing themselves as caregivers and the importance of taking care of themselves to take care of others.

Keywords: Caregivers; Primary Health Care; Medical Education; Complementary Therapies; Therapeutic Group.

1 Universidade Federal do Vale do São Francisco, Petrolina, Pernambuco, Brasil.

${ }^{2}$ Universidade Gama Filho, Rio de Janeiro, Rio de Janeiro, Brasil.

Editora-chefe: Rosiane Viana Zuza Diniz.

Editor associado: Danilo Borges Paulino.

Recebido em 14/09/2021; Aceito em 24/11/2021.

Avaliado pelo processo de double blind review. 


\section{INTRODUÇÃO}

Cuidado é amor, vínculo, zelo, atenção, dedicação, responsabilidade. Cuidar é uma atitude, é algo que está na constituição do ser humano e requer envolvimento afetivo com o outro'. A ação de cuidar é voluntária e complexa, visto que quem cuida pode também ter que lidar com sentimentos como raiva, medo, culpa, angústia, estresse, tristeza e irritação ${ }^{2-4}$. Com isso, a presença de tais emoções pode sinalizar o adoecimento do cuidador $2,5,6$.

Por definição, a sobrecarga do cuidador é o estado psicológico resultante da soma de desgaste físico, pressão emocional, isolamento social e demandas econômicas a partir da assistência a uma pessoa doente ${ }^{7,8}$. O tempo prolongado de adoecimento da pessoa cuidada, o elevado grau de dependência por auxilio, a falta de apoio social e um período de dedicação superior a três anos à pessoa enferma são fatores que influenciam negativamente na qualidade do cuidado prestado $0^{6,9-11}$.

No contexto da atenção primária à saúde (APS), é importante identificar a presença de estresse no cuidador. $\mathrm{O}$ reconhecimento de sinais de sobrecarga pode ser realizado a partir do acolhimento e da escuta atenta do cuidador durante a visita domiciliar à pessoa doente ${ }^{6,12}$. Diante de tal quadro, é imprescindível que opções sejam oferecidas às famílias e aos cuidadores, pois a finalidade de qualquer ação em saúde é o ato de cuidar. Em um contexto de sofrimento, o uso de tecnologias leves, como encontro, acolhimento, estabelecimento de vínculo, companheirismo, confiança e responsabilização pelo problema a ser enfrentado, pode ser suficiente para o restabelecimento do equilíbrio ${ }^{13}$.

Entre as intervenções para promover o cuidado a quem cuida na APS, temos o grupo terapêutico ${ }^{14}$. Tal recurso possibilita a troca de experiências e a transformação de práticas em saúde, a partir do encontro entre pessoas que compartilham as mesmas angústias e os profissionais que atuam na unidade de saúde e no Núcleo de Apoioà Saúde da Família (Nasf), no contexto doSistema Único de Saúde (SUS) ${ }^{14-16}$. Outro recurso acessível que se propõe a lançar um olhar holístico sobre o indivíduo, a comunidade e a natureza são as Práticas Integrativas e Complementares em Saúde (Pics) ${ }^{17}$. A potencialidade dessas práticas vem da lógica integrativa que compreende os aspectos da saúde e os entrelaça de forma multi, inter e transdisciplinar, acolhendo os saberes acadêmicos e comunitários no cuidado das pessoas ${ }^{18,19}$.

A estruturação de um grupo terapêutico com cuidadores sobrecarregados pelas demandas do cuidado em uma unidade de saúde do sertão foi motivada pelos seguintes fatores: 1. identificação de sofrimento nos cuidadores de pessoas acamadas atendidas em visitas domiciliares pela equipe da unidade de saúde; 2 . a grande vulnerabilidade social e econômica da maioria dos moradores do bairro Pedra Linda, na periferia da cidade de
Petrolina, em Pernambuco; 3. e a necessidade de estruturação de um projeto de intervenção como estratégia de aprendizado de duas alunas do período de internato do curso de Medicina da Universidade Federal do Vale do São Francisco (Univasf) durante o rodízio de Medicina da Família e Comunidade (MFC) na Estratégia Saúde da Família (ESF).

O curso de Medicina da Univasf é estruturado em seis anos, e, nos doisúltimos, o estudante vivencia o internato:estágio obrigatório em serviços de saúde conveniados à universidade. Essa estrutura visa ao aprendizado de técnicas necessárias à formação médica, sob supervisão de docentes ou preceptores nos serviços. No primeiro e no segundo ano do internato, o estudante atuará em períodos de oito a dez semanas, nas áreas de clínica médica, clínica cirúrgica, tocoginecologia, pediatria e MFC ${ }^{20}$. Para avaliação prática dos alunos no cenário de MFC, é proposto o desenvolvimento de um projeto de intervenção a partir da identificação de um problema observado no território. Tal instrumento é importante porque permite que o estudante pesquise e analise criticamente a realidade em que atua, assumindo compromissos e se responsabilizando pelas pessoas e pela comunidade.

O objetivo deste trabalho foi então relatar a experiência de estruturação de um grupo terapêutico com cuidadores informais que apresentavam sinais de sobrecarga e atuavam na prestação de cuidados a pessoas acamadas acompanhadas em visita domiciliar pela equipe de saúde, como projeto de intervenção de alunas do período de internato do curso de Medicina no rodízio de MFC na ESF. O grupo foi estruturado e desenvolvido em parceria com a médica residente do segundo ano do Programa de Residência em MFC, a preceptora do programa e a enfermeira da equipe na Unidade-Escola Josefa de Souza Silva, no bairro Pedra Linda, no sertão pernambucano.

\section{RELATO DE EXPERIÊNCIA}

Trata-se de estudo descritivo, tipo relato de experiência, realizado no período de maio a julho do ano de 2019. A detecção do sofrimento nos cuidadores informais como um problema foi percebido a partir da escuta atenta das alunas nas visitas domiciliares a pessoas acamadas realizadas com a equipe da ESF. A visita domiciliar é uma ferramenta de ensinoaprendizagem essencial à formação médica, pois possibilita vivenciar a integralidade do cuidado nos contextos individual e coletivo, além de permitir uma aproximação com os determinantes do processo saúde-doença no âmbito familiar ${ }^{21}$. Essa tecnologia permite trabalhar habilidades e atitudes como comunicação, observação, diálogo, relato oral e escrito, além de oportunizar a criação de espaços de subjetividade, nos quais é possível a identificação de uma rede de cuidados imprescindível para resolução dos problemas e continuidade da assistência ${ }^{22}$. 
A escolha do grupo terapêutico como estratégia de cuidado foi plausível a partir da identificação do quadro de sofrimento em mais de um cuidador acompanhado nas visitas domiciliares. Para que possa haver escuta e partilha de experiências, é recomendado que cada grupo tenha de três a 15 pessoas e que cada encontro tenha duração de 60, 90 ou 120 minutos, acontecendo de uma a três vezes por semana ${ }^{23}$. O planejamento das atividades e a definição dos objetivos a serem alcançados em cada encontro, assim como a listagem dos materiais e o orçamento, foram realizados pelas alunas e pela médica residente, levando em consideração o tempo de dez semanas que corresponde à duração do rodízio na ESF das estudantes do primeiro ano do internato de Medicina da Univasf.

Definiu-se também que, com base na importância do vínculo e do acolhimento para fortalecimento do grupo, as duas alunas, a médica residente, a preceptora do programa e a enfermeira da equipe tentariam estar presentes em todas as atividades, quando exerceriam a função de coordenadoras do grupo. Adotou-se o seguinte critério de inclusão: cuidadores informais com sinais de sobrecarga identificados pela escuta atenta da médica residente e das alunas de Medicina durante as visitas domiciliares da equipe às pessoas acamadas no território, sejam homens ou mulheres com idade acima de 18 anos. Não se definiram critérios de exclusão porque a simples presença, mesmo que por um único encontro, já significaria um êxito, visto que o cuidador estaria se permitindo receber cuidados por um momento da semana e que a falta poderia ocorrer em virtude das ocupações relacionadas à prestação de cuidado a si mesmo ou a outra pessoa.

O grupo terapêutico foi estruturado a partir da abordagem comunitária e de recursos como educação popular e as Pics, com a participação dos 12 cuidadores que aceitaram o convite - em um grupo fechado e heterogêneo. Os sete encontros ocorreram no espaço da própria unidade de saúde, no turno vespertino, uma vez por semana, com duração de 60 a 90 minutos. Cada encontro foi assim dividido: 1. acolhida, com a escuta da repercussão da atividade proposta no encontro anterior, 2. apresentação da atividade do dia com a vivência de uma prática de autocuidado e 3. finalização com a sugestão de uma tarefa a ser desenvolvida em casa, durante a semana, até o próximo encontro. O processo de avaliação de cada participante foi realizado a partir da escuta atenta das reverberações das atividades propostas nos encontros e nas visitas domiciliares. A estruturação de cada uma dessas atividades está descrita no Quadro 1.

No primeiro encontro, foi realizada a escuta de cada participante, que respondeu às perguntas disparadoras: "Quem

Quadro 1. Planejamento de atividades - grupo dos cuidadores, Petrolina, Pernambuco, 2019.

\begin{tabular}{|c|c|c|c|}
\hline & ATIVIDADE/OBJETIVOS & RECURSOS & ATIVIDADE PARA CASA \\
\hline ENCONTRO SEMANA 1 & $\begin{array}{l}\text { Acolhimento dos cuidadores. } \\
\text { Responder às perguntas: "Quem } \\
\text { é você?" e "Como você se sente } \\
\text { quando cuida de alguém?". }\end{array}$ & $\begin{array}{l}\text { Arteterapia utilizando leitura de } \\
\text { livros infantis (biblioterapia), uso } \\
\text { de aromas para o ambiente e } \\
\text { reflexologia (auriculoterapia). }\end{array}$ & $\begin{array}{l}\text { Dedicar a si mesmo dez } \\
\text { minutos do dia. }\end{array}$ \\
\hline ENCONTRO SEMANA 2 & $\begin{array}{l}\text { Acolhimento dos cuidadores. } \\
\text { Responder à pergunta: "Como } \\
\text { você lida com os estresses do dia } \\
\text { a dia?". }\end{array}$ & $\begin{array}{l}\text { Arteterapia utilizando } \\
\text { jardinagem e reflexologia } \\
\text { (auriculoterapia). }\end{array}$ & $\begin{array}{l}\text { Cultivar/contemplar algo que } \\
\text { permita sentir felicidade (como } \\
\text { uma planta). }\end{array}$ \\
\hline ENCONTRO SEMANA 3 & $\begin{array}{l}\text { Acolhimento dos cuidadores. } \\
\text { Responder à pergunta: "A quem } \\
\text { posso pedir ajuda?". }\end{array}$ & $\begin{array}{l}\text { Construção do genograma e } \\
\text { ecomapa, e uso de aromas para o } \\
\text { ambiente. }\end{array}$ & $\begin{array}{l}\text { Exercitar pedir ajuda e tirar um } \\
\text { turno de folga. }\end{array}$ \\
\hline ENCONTRO SEMANA 4 & $\begin{array}{l}\text { Acolhimento dos cuidadores. } \\
\text { Reconhecer os direitos dos } \\
\text { cuidadores. }\end{array}$ & $\begin{array}{l}\text { Arteterapia utilizando jogos e } \\
\text { músicas, uso de aromas para } \\
\text { o ambiente e reflexologia } \\
\text { (auriculoterapia). }\end{array}$ & $\begin{array}{l}\text { Exercitar pedir ajuda e tirar um } \\
\text { dia de folga. }\end{array}$ \\
\hline ENCONTRO SEMANA 5 & $\begin{array}{l}\text { Acolhimento dos cuidadores. } \\
\text { Responder à pergunta: "Como eu } \\
\text { me cuido?". }\end{array}$ & $\begin{array}{l}\text { Arteterapia utilizando jogos e } \\
\text { músicas, e uso de aromas para o } \\
\text { ambiente. }\end{array}$ & $\begin{array}{l}\text { Refletir sobre os desejos para } \\
\text { o tempo presente e os planos } \\
\text { para o futuro. }\end{array}$ \\
\hline ENCONTRO SEMANA 6 & $\begin{array}{l}\text { Acolhimento dos cuidadores. } \\
\text { Responder à pergunta:"Como } \\
\text { me preparar para cuidar de } \\
\text { alguém?". }\end{array}$ & $\begin{array}{l}\text { Para o cenário, macas, } \\
\text { colchonetes, travesseiros } \\
\text { e cadeira de roda. Material } \\
\text { impresso. }\end{array}$ & $\begin{array}{l}\text { Exercitar os alongamentos } \\
\text { antes, durante e ao final do dia. }\end{array}$ \\
\hline ENCONTRO SEMANA 7 & $\begin{array}{l}\text { Acolhimento dos cuidadores } \\
\text { e inauguração do "Jardim do } \\
\text { Cuidado". }\end{array}$ & $\begin{array}{l}\text { Colchonetes e espelhos. } \\
\text { Massoterapia, meditação, uso } \\
\text { de aromas para o ambiente, } \\
\text { reflexologia (auriculoterapia) e } \\
\text { arteterapia a partir da jardinagem. }\end{array}$ & $\begin{array}{l}\text { Fortalecer a rede de cuidados } \\
\text { pedindo ajuda e contando } \\
\text { com a ajuda dos familiares, do } \\
\text { grupo e da equipe da unidade } \\
\text { de saúde. }\end{array}$ \\
\hline
\end{tabular}

Fonte: Elaborado pelas autoras. 
é você?" e "Como você se sente quando cuida de alguém?". Em seguida, utilizando biblioterapia, fez-se a leitura do livro A fada afilhada, de Márcio Vassallo, que salienta de forma lúdica o impacto da sobrecarga de cuidados no cuidador e a importância do autocuidado. Como propostas de atividades a serem experimentadas durante a semana até o próximo encontro, sugeriram-se exercícios, automassagens e chás de plantas medicinais.

Na segunda reunião, abordaram-se aspectos relacionados a como os cuidadores lidam com o estresse diário. Questionou-se se havia alguma atividade do dia a dia deles em que se sentiam mais sobrecarregados e o que geralmente faziam para que pudessem ficar bem. A proposta do dia foi trabalhar arteterapia utilizando a jardinagem como um recurso terapêutico para lidar com o estresse. Foram levadas mudas de plantas, e cada participante se disponibilizou a cultivá-las. A fim de expandirem o olhar para o espaço físico da unidade de saúde, os cuidadores resolveram transformar um canteiro entre os consultórios que era morto e sem função, a cada encontro e de forma coletiva, em um jardim. A partir desse encontro, todas as reuniões começaram com a escuta dos participantes de como haviam ressoado o encontro anterior e a atividade sugerida para a semana.

O terceiro encontro foi o momento de conhecer a família e a rede social dos cuidadores, a partir da construção do genograma e do ecomapa. Tais instrumentos são utilizados na abordagem familiar como formas eficientes de colher informações: a partir do genograma, é possível obter dados sobre a constituição familiar - estrutura, composição, padrões e relacionamentos -, permitindo, com sua análise, ter uma percepção da situação psicossocial do usuário e dos familiares ${ }^{24}$. O ecomapa sinaliza os sistemas que circundam a pessoa, a família e a comunidade e que se conectam com elas ${ }^{25}$. Saber como utilizar tais ferramentas é essencial na formação médica, pois elas colaboram para abordagem sistêmica do indivíduo. Com tais recursos, foi possível aos cuidadores descobrir quem e onde procurar ajuda e perceber que não estavam sozinhos. $A$ atividade sugerida para casa foi se permitir pedir e aceitar ajuda para o cuidado do outro.

$\mathrm{Na}$ quarta reunião com o grupo, foram trabalhados os direitos do cuidador, ressaltando que indivíduos que se cuidam apresentam melhores condições emocionais e físicas de zelar por outras pessoas ${ }^{26}$. Também foi resolvida com o grupo a dificuldade de uma das integrantes em organizar as medicações da pessoa por ela cuidada. As alunas organizaram vasilhas sinalizadas com figuras (manhã = sol; tarde $=$ prato de almoço; noite = lua e estrelas), e cada cartela de comprimidos foi assinalada com diferentes cores e distribuída na vasilha correspondente ao horário de uso. Registraram-se as informações sobre horário de medicamentos em uma folha A4, e a cuidadora foi orientada a fixar a folha em um local visível, que pudesse ser consultada, quando necessário, por qualquer pessoa que estivesse auxiliando no cuidado.

Para o quinto encontro, convidamos uma psicóloga para conversar sobre autocuidado. Foram escritas em papéis perguntas disparadoras como: "O que você faz para cuidar de si?", "Como você se vê daqui a cinco anos?" e "Qual o seu maior sonho?". Colocaram-se essas perguntas numa caixinha que passou de mão em mão entre as participantes, em um jogo tipo "batata quente". Para finalização da atividade, fez-se o acolhimento com uma roda de abraços.

No sexto encontro, convidamos a fisioterapeuta do Nasf para conversar com os cuidadores sobre como lidar com situações do dia a dia que causam dor no corpo, como dar banho e movimentar a pessoa no leito. Os cuidadores receberam orientações sobre exercícios de alongamento. Em seguida, com a utilização de macas, colchonetes, travesseiros e cadeira de rodas, os cuidadores receberam informações sobre os seguintes procedimentos: acomodar a pessoa cuidada na cama, adotar mudanças de decúbito, colocar a pessoa sentada, transportála da cama para a cadeira e ajudá-la a caminhar. Por fim, cada cuidador recebeu um material impresso com as orientações.

No sétimo encontro, organizou-se o encerramento do grupo. Em uma das salas, posicionaram-se colchonetes e cadeiras, e os cuidadores (que quisessem) foram orientados a deitar ou sentar para que pudessem receber alguns cuidados, como massoterapia, aromaterapia e auriculoterapia. Distribuíram-se pequenos espelhos para que cada um se olhasse e reconhecesse aquela pessoa que estava aprendendo a cuidar de si. Em seguida, foi dado a cada um o certificado de "Melhor Cuidador do Mundo". Após esse momento, todos os participantes foram convidados para inaugurar o "Jardim do Cuidado", resultado da transformação individual e coletiva dos participantes do grupo terapêutico que transbordou "o cuidado" para o espaço físico da unidade de saúde.

\section{DISCUSSÃO}

Cada um dos encontros relatados possibilitou reflexões, mudanças de hábitos e o treinamento de habilidades. Já no primeiro, os participantes puderam perceber que não estavam sozinhos em relação às queixas: o sofrimento de um se encontrava no outro. No grupo, o que perceberam como diferente foi o tempo que cada um executava a função de cuidador e o grau de autonomia da pessoa que recebia os cuidados. Um trabalho com 14 cuidadores de idosos acompanhados em uma unidade de saúde de Porto Alegre concluiu que quanto maior é o grau de dependência da pessoa em relação ao cuidador, maior é a sobrecarga deste ${ }^{8}$. Nesse contexto, o tempo prolongado dedicado ao zelo de 
outra pessoa ou a perspectiva desse tempo, além do quadro de isolamento no gerenciamento das demandas, contribuíam também para aumentar o sofrimento do cuidador ${ }^{27}$.

Em cada uma das atividades, as alunas puderam exercitar a gestão do tempo e executar a função de mediadoras na coordenação do grupo. A partir dos discursos dos cuidadores, elas perceberam a importância de ser espaço de escuta para cada indivíduo, compreendendo-o integralmente, atitude imprescindível para estabelecimento do vínculo e relação médico-paciente. Também puderam desconstruir a imagem do médico como prescritor, vivenciando o cuidar do outro a partir de tecnologias leves, como a leitura de um livro infantil.

Foi interessante perceber que cada cuidador estava se organizando para estar presente nas reuniões, pois se sentia acolhido e identificava o benefício dos momentos de autocuidado, mesmo que experimentados só alguns minutos por dia. Igualmente significativo foi observar o autorreconhecimento das alunas e profissionais da equipe como cuidadores que estavam se permitindo experimentar cada atividade de autocuidado proposta para o grupo. Cuidar integralmente das pessoas só faz sentido se o cuidador cuidar de si mesmo: só é possível olhar para o sofrimento do outro se a pessoa estiver cuidando do próprio sofrimento ${ }^{28}$.

O espaço também foi importante para discutir com as estudantes conceitos básicos como letramento funcional em saúde, que consiste no entendimento, na interpretação e na aplicação de informações sobre saúde, para ações preventivas, partindo da compreensão de que pessoas com um nível de letramento satisfatório conseguem se cuidar melhor que aquelas com um nível limitado ${ }^{29}$. Isso revela a importância de os profissionais de saúde se preocuparem com a cognição das pessoas atendidas, avaliando a necessidade de adequações para garantir a assimilação das orientações.

A repercussão do encontro com a fisioterapeuta foi visualizada nas visitas domiciliares. Em algumas residências, o material impresso estava bem próximo ao leito da pessoa cuidada e era consultado principalmente para os alongamentos das que estavam acamadas. Foi observada uma maior destreza para movimentação da pessoa no leito. Um estudo realizado com 20 cuidadores informais de pessoas com disfunções decorrentes de acidente vascular cerebral ou de trauma raquimedular acompanhadas em uma clínica do Paraná evidenciou que dar orientações fisioterapêuticas aos cuidadores, apesar de não encontrar diferença significativa em relação à sobrecarga ou ao acréscimo geral na qualidade de vida, repercute significativamente na redução da dor e na melhora da saúde mental dos cuidadores ${ }^{30}$.

Na finalização do grupo, foi possível perceber que os participantes estavam mais abertos a receber cuidados. $\mathrm{O}$ contato com algumas Pics permitiu melhorar a percepção sobre os impactos do estresse no corpo, em forma de dores e tensões. Aplicadas na medicina convencional, as Pics possibilitaram uma abordagem menos invasiva e propiciaram a compreensão do adoecimento. Quando os participantes assimilaram que, para cuidar do outro, é preciso cuidar de si mesmos, ficou mais leve oferecer os cuidados. E o jardim floresceu e foi inaugurado.

O outro jardim desse trabalho foi o aprendizado das estudantes em relação ao próprio autocuidado e as possibilidades de intervenção em uma comunidade. Na execução do grupo terapêutico, elas se permitiram conhecer, experimentar e aplicar as Pics, bem como acompanhar os resultados a partir dos relatos dos cuidadores. Puderam ainda vivenciar o estabelecimento da relação médico-paciente a partir do vínculo e a prescrição de outros cuidados com base na escuta centrada na pessoa, contribuindo para a não medicalização de problemas como o sofrimento humano. Compreenderam a importância do trabalho em equipe e aprenderam a coordenar o grupo e fazer a gestão do tempo. Além disso, entenderam o significado de equidade, integralidade e longitudinalidade na prática da MFC, e, por conta disso, floresceram.

\section{CONCLUSÃO}

Cuidar é uma prática de respeito e compaixão, um movimento de dar e receber, de pedir e aceitar. A diminuição do estresse, da sobrecarga e do isolamento dos cuidadores das pessoas acompanhadas nas visitas domiciliares foi possível a partir do uso de tecnologias leves trabalhadas no grupo terapêutico. A identificação e o fortalecimento da rede de apoio representaram um processo fundamental para a autonomia dos cuidadores na promoção da assistência, assim como o reconhecimento do autocuidado como um processo contínuo e de responsabilidade do cuidador e da equipe de saúde.

Percebeu-se que o grupo existia fora da unidade de saúde, em encontros no final da tarde ou da semana para compartilhar o prazer de viver. Apesar disso, e talvez pela estruturação do grupo com frequência semanal, alguns participantes tiveram dificuldade de estar presentes nos encontros. Alguns porque a pessoa que estava sendo cuidada precisou ficar internada ou por não terem conseguido alguém para cuidar no horário programado para reunião. Um intervalo de tempo maior entre os encontros pode melhorar a assiduidade dos participantes.

A importância deste relato foi perceber a contribuição de uma intervenção na comunidade também para formação das duas alunas do período de internato do curso de Medicina. Foi relevante demonstrar que para cuidar de gente é preciso ensinar e aprender a escutar, respeitar o conhecimento e as escolhas do outro e que essas práticas são possíveis no riquíssimo cenário da APS, na ESF e no rodízio de MFC. Além 
disso, são experiências possíveis de ser reproduzidas em outras unidades de saúde, abordando outros temas, de modo a colaborar significativamente na formação de estudantes e na vida dos profissionais e usuários que frequentam os serviços.

\section{CONTRIBUIÇÃO DAS AUTORAS}

Ana Vitória Teixeira de Medeiros Gomes e Erika Denise de Vasconcelos Florentino participaram da estruturação e coordenação do projeto de intervenção na UBS, do planejamento, da construção científica e da execução do projeto, e da elaboração e redação final do manuscrito. Myrlla Soares de Oliveira, Thaiany Rego Silva e Jucimara Alves de Souza participaram da estruturação e coordenação do projeto de intervenção na UBS, e da revisão final do manuscrito.

\section{CONFLITO DE INTERESSES}

Declaramos não haver conflito de interesses.

\section{FINANCIAMENTO}

Declaramos não haver financiamento.

\section{REFERÊNCIAS}

1. Boff L. Saber cuidar: ética do humano - compaixão pela terra. 20a ed. Petrópolis: Vozes; 2014.

2. Brandão FSR, Costa BGS, Cavalcanti ZR, Bezerra MR, Alencar LCA, Leal MCC. Sobrecarga dos cuidadores idosos assistidos por um serviço de atenção domiciliar. Rev Enferm UFPE On Line. 2017;11(supl 1):S272-9. doi: 10.5205/ reuol.7995-69931-4-SM.1101sup201704.

3. Baptista BO, Beuter M, Girardon-Perlini NMO, Brondani CM, Budó MLD, Santos NO. A sobrecarga do familiar cuidador no âmbito domiciliar: uma revisão integrativa da literatura. Rev Gaucha Enferm. 2012;33(1):147-56. doi: 10.1590/S1983-14472012000100020.

4. Ahnerth NMS, Dourado DM, Gonzaga NM, Rolim JA, Batista EC. "A gente fica doente também": percepção do cuidador familiar sobre o seu adoecimento. Gerais: Rev Interinst Psicol. 2020;13(1):1-20. doi: 10.36298/ gerais2020130106.

5. Jesus ITM, Orlandi AAS, Zazzetta MS. Sobrecarga, perfil e cuidado: cuidadores de idosos em vulnerabilidade social. Rev Bras Geriatr Gerontol. 2018;21(2):199-209. doi: 10.1590/1981-22562018021.170155.

6. Mahmud SJ, Mano MAM, Lopes JMC, Savassi LCM. Abordagem comunitária: cuidado domiciliar. In: Gusso G, Lopes JMC, Dias LC, organizadores. Tratado de medicina e comunidade: princípios, formação e prática. 2a ed. Porto Alegre: Artmed; 2019. v. 1, p. 313-24.

7. Rezende G, Gomes CA, Rugno FC, Carvalho RC, De Carlo MMRP. Sobrecarga de cuidadores de pessoas em cuidados paliativos: revisão integrativa da literatura. Medicina (Ribeirão Preto). 2016;49(4):344-54. doi: 10.11606/ issn.2176-7262.v49i4p344-354.

8. Lopes CC, Oliveira GA, Stigger FS, Lemos AT. Associação entre a ocorrência de dor e sobrecarga em cuidadores principais e o nível de independência de idosos nas atividades de vida diária: estudo transversal. Cad Saude Colet. 2020;28(1):98-106. doi: 10.1590/1414-462X202028010184.

9. Drulla AG, Alexandre AMC, Rubel FI, Mazza VA. A visita domiciliar como ferramenta ao cuidado familiar. Cogit Enferm. 2009;14(4):667-74. doi: http://dx.doi.org/10.5380/ce.v14i4.16380.

10. Rezende TCB, Coimbra AMV, Costallat LTL, Coimbra IB. Factors of high impacts on the life of caregivers of disabled elderly. Arch Gerontol Geriatr. 2010;51(1):76-80. doi: 10.1016/j.archger.2009.08.003.
11. Lino VTS, Rodrigues NCP, Camacho LAB, O'Dwyer G, Lima IS, Andrade $M K N$, et al. Prevalência de sobrecarga e respectivos fatores associados em cuidadores de idosos dependentes, em uma região pobre do Rio de Janeiro, Brasil. Cad Saude Publica. 2016;32(6):e00060115. doi: 10.1590/0102-311X00060115.

12. Faria AA, Aparecido AM, Cruz GL, Khater E. Cuidando de quem cuida - o papel do psicólogo com cuidadores de pacientes paliativos. Saúde Foco. 2017];1(9):25-36 [acesso em 2 set 2020]. Disponível em: https://portal. unisepe.com.br/unifia/wpcontent/uploads/sites/10001/2018/06/004_ artigo_saude_template.pdf.

13. Merhy EE. A perda da dimensão cuidadora na produção da saúde - uma discussão do modelo assistencial e da intervenção no seu modo de trabalhar a assistência. In: Reis AT, Santos AF, Campos CR, Malta DC, Merhy EE, editores. Sistema Único de Saúde em Belo Horizonte: reescrevendo o público. São Paulo:Xamã; 1998. p. 103-20 [acesso em 2 de set. 2020]. Disponível em: https:// www.researchgate.net/publication/33023409_A_perda_da_dimensao_ cuidadora_na_producao_da_saude_uma_discussao_do_modelo_ assistencial_e_da_intervencao_no_seu_modo_de_trabalhar_a_assistencia.

14. Casanova F, Osorio LC, Dias LC. Abordagem comunitária: grupos na atenção primária à saúde. In: Gusso G, Lopes JMC, Dias LC, organizadores. Tratado de medicina e comunidade: princípios, formação e prática. 2a ed. Porto Alegre: Artmed; 2019. v. 1, p. 325-33.

15. Santos RL, Sousa MFB, Brasil D, Dourado M. Intervenções de grupo para sobrecarga de cuidadores de pacientes com demência: uma revisão sistemática. Rev Psiquiatr Clín. 2011;38(4): 161-7. doi: 10.1590/S010160832011000400009

16. Oliveira KS, Baduy RS, Melchior R. O encontro entre Núcleo de Apoio à Saúde da Família e as equipes de Saúde da Família: a produção de um coletivo cuidador. Physis. 2019;29(4):e290403. doi: 10.1590/s010373312019290403.

17. Brasil. Portaria no 849 , de 27 de março de 2017. Inclui a arteterapia ayurveda, biodança, dança circular, meditação, musicoterapia, naturopatia, osteopatia, quiropraxia, reflexoterapia, reiki, shantala, terapia comunitária integrativa e yoga à Política Nacional de Práticas Integrativas e Complementares. Diário Oficial da União; 28 mar 2017.

18. Barreto AF. Práticas Integrativas e Complementares como ética da sensibilidade no cuidado humano. J Manag Prim Heal Care. 2017;8(2):181202. doi: https://doi.org/10.14295/jmphc.v8i2.525.

19. Yaari M, Roman AC. Práticas integrativas. In: Gusso G, Lopes JMC, Dias LC, organizadores. Tratado de medicina e comunidade: princípios, formação e prática. 2a ed. Porto Alegre: Artmed; 2019. v. 1, p. 786-97.

20. Universidade Federal do Vale do São Francisco. Colegiado Acadêmico de Medicina - Petrolina/PE. Regimento do internato médico. 2019 [acesso em 12 set 2020]. Disponível em: http://www.medicina.univasf.edu.br/ arquivos/Regimento\%20do\%20Internato\%20Medico.pdf.

21. Romanholi RMZ, Cyrino EG. A visita domiciliar na formação de médicos: da concepção ao desafio do fazer. Interface Comun Saúde Educ. 2012;16(42):693-705. doi: 10.1590/S1414-32832012000300009.

22. Lopes WO, Saupe R, Massaroli A. Visita domiciliar: tecnologia para o cuidado, o ensino e a pesquisa. Cienc Cuid Saúde. 2008;7(2):241-7. doi: 10.4025/cienccuidsaude.v7i2.5012

23. Osório LC. Grupoterapias: abordagens atuais. Porto Alegre: Artmed; 2007

24. Muniz JR, Eisenstein E. Genograma: informações sobre família na (in) formação médica. Rev Bras Educ Med. 2009;33(1):72-9. doi: https://doi. org/10.1590/S0100-55022009000100010.

25. Dias LC. Abordagem familiar. In: Gusso G, Lopes JMC, Dias LC, organizadores. Tratado de medicina e comunidade: princípios, formação e prática. 2a ed. Porto Alegre: Artmed; 2019. v. 1, p. 282-92.

26. Born $\mathrm{T}$, organizadora. Cuidar melhor e evitar a violência: manual do cuidador da pessoa idosa Brasília: Secretaria Especial dos Direitos Humanos, Subsecretaria de Promoção e Defesa dos Direitos Humanos; 2008 [acesso em 3 set 2020]. Disponível em: https://bibliotecadigital.mdh.gov.br/ jspui/handle/192/456\#: :text=O\%20manualser\%C3\%A1\%20um\%20 instrumento\%20que,lhe\%20um\%20envelhecer\%20com\%20dignidade.

27. Fernandes MGM, Garcia TR. Determinantes da tensão do cuidador familiar de idosos dependentes. Rev Bras Enferm. 2009;62(1):57-63. doi: http:// dx.doi.org/10.1590/S0034-71672009000100009.

28. Arantes ACQ. A morte é um dia que vale a pena viver. Rio de Janeiro: Casa da Palavra; 2016. 
29. Passamai MPB, Sampaio HAC, Dias AMI, Cabral LA. Letramento funcional em saúde: reflexões e conceitos sobre seu impacto na interação entre usuários, profissionais e sistema de saúde. Interface Comun Saúde Educ. 2012;16:301-14 [acesso em 12 set 2020]. Disponível em: scielo. br/pdf/icse/2012nahead/aop2812\#: :text=Functional\%20health\%20 literacy.de\%20decisões\%20adequadas\%20em\%20saúde.
30. Fernandes $B C W$, Ferreira $K C P$, Marodin MF, Val MON, Fréz AR. Influência das orientações fisioterapêuticas na qualidade de vida e na sobrecarga de cuidadores. Fisioter Mov. 2013;26(1): 151-8. doi: http://dx.doi. org/10.1590/S0103-51502013000100017. 